\title{
Erratum to: A general result on the uniform in bandwidth consistency of kernel-type function estimators
}

\author{
David M. Mason • Jan W. H. Swanepoel
}

Published online: 25 November 2014

(C) Sociedad de Estadística e Investigación Operativa 2014

\section{Erratum to: Test (2011) 20:72-94 DOI 10.1007/s11749-010-0188-0}

We want to point out the following corrections to the original article:

1. Replace " $(\log n)^{-1}$ " by " $c \log n / n$ " in their Corollaries $1-3$ and three lines above Eq. (2.16).

2. In Eq. (2.5) replace " $O\left(\sqrt{b_{n}}\right)$ " by " $=O\left(\sqrt{b_{n}\left(\frac{\left|\log b_{n}\right|}{\log \log n} \vee 1\right)}\right)=o(1) "$.

3. In Eqs. (2.6) and (2.7) and in the two lines below their Corollary 2, replace “ $\sqrt{b_{n} \log \log n}$ ” by “ $\sqrt{b_{n}\left(\log \log n \vee\left|\log b_{n}\right|\right)}$ ”.

4. Three lines below Eq. (2.15) replace" $0<b_{n}<1$ satisfying $b_{n} \geq(\log n)^{-1}$ and $\sqrt{n} b_{n} / \sqrt{\log \log n}=o(1)$ " by" $b_{n}$ satisfying $b_{n} \geq c \log n / n$ and $b_{n} \rightarrow 0$ ".

5. In (F.ii) given in the Theorem, $\mathcal{G}$ should be $\mathcal{G}_{\gamma}$.

For more details of corrections 1-4 refer to Corollaries 1.12, 1.13 and 1.14 in Mason and Swanepoel (2013).

The online version of the original article can be found under doi:10.1007/s11749-010-0188-0.

D. M. Mason ( $\square)$

Department of Applied Economics and Statistics, University of Delaware, Newark, DE 19716, USA e-mail:davidm@udel.edu

J. W. H. Swanepoel

Department of Statistics, North-West University, Potchefstroom, South Africa 


\section{Reference}

Mason DM, Swanepoel JWH (2013) Uniform in bandwidth limit laws for kernel distribution function estimators. In: Banerjee M, Bunea F, Huang J, Koltchinskii V, Maathuis MH (eds) IMS collections: probability to statistics and back: high-dimensional models and processes-a Festschrift in Honor of Jon A. Wellner, vol 9, pp 241-253 